\title{
FIELDS AND ELEMENTARY PARTICLES
}

\section{NONCOMMUTATIVE GAUGE THEORIES}

\section{WOHLGENANNT}

We review two different noncommutative gauge models generalizing the approaches which lead to renormalizable scalar quantum field theories. One of them implements the crucial IR damping of the gauge field propagator in the so-called "soft breaking" part. We discuss one-loop renormalizability.

\section{Introduction}

There are various motivations for studying noncommutative geometries. They range from general considerations in Quantum Field Theory (QFT) $[1,2]$ and (Quantum) Gravity [3,4] to String Theory and Matrix Models [5-7] and purely mathematical considerations [8]. One of the first applications of noncommutative ideas was already within the realm of gauge theories, namely the Quantum Hall effect [9]. What is most remarkable, in my eyes, is the intimate connection between noncommutative gauge theory and gravity. This connection is not fully understood at present and studied from different points of view, see, e.g., [10-15] and references therein for a merely exemplary list of quotations.

In this note, we concentrate on models for noncommutative gauge theories, where the idea of renormalizability will be a guiding principle. Furthermore, we consider the canonically deformed 4D Euclidean space. The coordinates satisfy the commutation relations

$\left[x^{i}, x^{j}\right]=\mathrm{i} \Theta^{i j}$,

where $\Theta^{i j}=-\Theta^{j i}=$ const, and the star product is given by the Moyal-Weyl product,

$f \star g(x)=\left.\mathrm{e}^{\frac{\mathrm{i}}{2} \Theta^{i j} \partial_{i}^{x} \partial_{j}^{y}} f(x) g(y)\right|_{y \rightarrow x}$.

In the next section, we will discuss the so-called $\mathrm{UV} / \mathrm{IR}$ mixing problem in the case of scalar field theory. It is a thread to renormalizability. Up to now, there are two different models, which overcome this problem and which are perturbatively renormalizable to all orders. Both are formulated on the canonically deformed Euclidean space. In Section 3, we will attempt to generalize both approaches to noncommutative $U(1)$ gauge theory. A brief summary and some concluding remarks follow in Section 4.

\section{UV/IR Mixing in Scalar Theories}

The simplest approach to the noncommutative $\phi^{4}$ theory is to take the commutative action and to replace the pointwise products by star products. Since the star product is not relevant for bilinear expressions, only the self-interaction term is modified, and we obtain

$S=\int d^{4} x\left(\frac{1}{2} \partial_{\mu} \phi \partial_{\mu} \phi+\frac{m^{2}}{2} \phi^{2}+\frac{\lambda}{4 !} \phi \star \phi \star \phi \star \phi\right)$.

The above action determines the Feynman rules. The propagator is the same as in the commutative case,

$G(p)=\frac{1}{p^{2}+m^{2}}$,

while the vertex is decorated by momentum dependent phase factors:

$\Gamma\left(p_{1}, \ldots, p_{4}\right)=-\lambda \delta^{(4)}\left(p_{1}+p_{2}+p_{3}+p_{4}\right) \mathrm{e}^{-\mathrm{i} \sum_{i<j} p_{i} \Theta p_{j}}$.

As a consequence, new types of Feynman graphs occur: In addition to the ones known from the commutative space, where no phases depending on internal loop momenta and showing the usual UV divergences appear, the so-called nonplanar graphs, which are regularized by phases depending on internal momenta, come into the 
game. One-loop calculations have been performed explicitly [16-20], and, hence, the UV/IR mixing problem has been found: Due to the phases in the nonplanar graphs, their UV sector is regularized, on the one hand, but, on the other hand, this regularization implies divergences for small external momenta. For example, let us consider the two-point tadpole graph. It is given by the expression

$$
\Pi(\Lambda, p) \propto \lambda \int d^{4} k \frac{2+\cos (k \tilde{p})}{k^{2}+m^{2}}=\Pi^{U V}(\Lambda)+\Pi^{I R}(\Lambda, p) .
$$

The planar contribution is, as usual, quadratically divergent in the UV cutoff $\Lambda$, i.e. $\Pi^{U V} \sim \Lambda^{2}$, and the nonplanar part is regularized by the cosine,

$\Pi^{I R} \sim \frac{1}{\tilde{p}^{2}}$

where $\tilde{p}_{\mu}=\Theta_{\mu \nu} p_{\nu}$. The original UV divergence is not present, but reappears when $\tilde{p} \rightarrow 0$ representing a new kind of infrared divergence. Since both divergences are related to each other, one speaks of "UV/IR mixing". At the one-loop level, this is no problem through. It corresponds to a counter term

$\int d^{4} p \tilde{\phi}(p) \frac{1}{\tilde{p}^{2}} \tilde{\phi}(-p)$,

which is well behaved even in the limit $\tilde{p} \rightarrow 0$. But the higher loop insertions then lead to a term of the form

$\int d^{4} p \tilde{\phi}(p) \frac{1}{\left(\tilde{p}^{2}\right)^{n}} \tilde{\phi}(-p)$,

where $n$ is the number of insertions. Clearly, this term exhibits a serious IR singularity. It is this mixing which renders the action (3) nonrenormalizable. Two different strategies to cure UV/IR mixing are known. Both modify the propagator by adding an additional term quadratic in the fields: An oscillator term (Section 2 2.1) and a $1 / \tilde{p}^{2}$-term (Section 22.2 ), respectively. In what follows, we will briefly review those approaches.

\subsection{The scalar Grosse-Wulkenhaar model}

After adding an oscillator potential and some awkward rewritting, action (3) becomes [21, 22]

$$
\begin{aligned}
& S=\int d^{4} x\left(\frac{1}{2} \phi \star\left[\tilde{x}_{\nu},\left[\tilde{x}^{\nu}, \phi\right]\right]+\right. \\
& \left.\left.+\frac{\Omega^{2}}{2} \phi \star\left\{\tilde{x}_{\nu}, \tilde{x}^{\nu}, \phi\right]\right\}+\frac{\mu^{2}}{2} \phi \star \phi+\frac{\lambda}{4 !} \phi \star \phi \star \phi \star \phi\right),
\end{aligned}
$$

where $\tilde{x}_{\nu}=\theta_{\nu \alpha}^{-1} x^{\alpha}$, and we have used i $\partial_{\mu} \mathrm{f}=\left[\tilde{\mathrm{x}}_{\mu}, \mathrm{f}\right]$. This action is covariant, i.e.

$S[\phi ; \mu, \lambda, \Omega] \mapsto \Omega^{2} S\left[\phi ; \frac{\mu}{\Omega}, \frac{\lambda}{\Omega^{2}}, \frac{1}{\Omega}\right]$,

under the so-called Langmann-Szabo duality transformation [23] between position and momentum:

$\hat{\phi}(p) \longleftrightarrow \pi^{2} \sqrt{|\operatorname{det} \Theta|} \phi(x), \quad p_{\mu} \longleftrightarrow 2 \tilde{x}_{\mu}$,

where $\hat{\phi}\left(p_{a}\right)=\int d^{4} x_{a} \mathrm{e}^{(-1)^{\mathrm{a}} \mathrm{ip}_{\mathrm{a}, \mu} \mathrm{x}_{\mathrm{a}, \mu}} \phi\left(\mathrm{x}_{\mathrm{a}}\right)$. The index $a$ is labelling the legs of vertex and propagator, respectively, and defines the direction of the corresponding momentum. This becomes a symmetry at $\Omega=1$. Due to the oscillator term, the propagator is modified, and an IR damping is implemented. The propagator is given by the Mehler kernel:

$K_{M}(p, q)=\frac{\omega^{3}}{8 \pi^{2}} \int_{0}^{\infty} \frac{d \alpha}{\sinh ^{2} \alpha} \times$

$\times \mathrm{e}^{-\frac{\omega}{4}(p-q)^{2} \operatorname{coth} \frac{\alpha}{2}-\frac{\omega}{4}(p+q)^{2} \tanh \frac{\alpha}{2}}$,

where $\omega=\Theta / \Omega$. The IR damping is also responsible for a proper handling of the UV/IR mixing problem. The model is renormalizable to all orders in perturbation theory. The propagator depends on two momenta, an incoming and an outgoing momentum, since the explicit $x$-dependence of the action breaks the translation invariance. Therefore, the momentum conservation is broken as well. Remarkably, the oscillator term can be interpreted as a coupling of the scalar field to the curvature of some specific noncommutative background [24].

\section{2. $1 / p^{2}$ model}

In the second approach, a nonlocal term is added to action (3). In the momentum space, it reads [25]

$S_{n l}=\int d^{4} p \frac{a}{2} \tilde{\phi}(p) \frac{1}{\tilde{p}^{2}} \tilde{\phi}(-p)$.

This is exactly the counter term (8) we have discussed before. The resulting action is translation-invariant, and thus the momentum conservation holds. Term (14) implements the IR damping for the propagator, i.e. $G(p) \rightarrow 0$ as $p \rightarrow 0$. The modified propagator has the form

$G(p)=\frac{1}{p^{2}+m^{2}+\frac{a^{2}}{p^{2}}}$. 
The damping effect of the propagator becomes obvious, when one considers higher-loop orders. An $n$-fold insertion of the divergent one-loop result (7) into a single large loop can be written as

$\Pi^{n \mathrm{np}-\mathrm{ins} .}(p) \approx \lambda^{2} \int d^{4} k \frac{\mathrm{e}^{\mathrm{ik} \tilde{\mathrm{p}}}}{\left(\tilde{k}^{2}\right)^{n}\left[k^{2}+m^{2}+\frac{a^{\prime 2}}{k^{2}}\right]^{n+1}}$,

neglecting any effects due to the recursive renormalization and approximating the insertions of irregular single loops by the most divergent (quadratic) IR divergence. For model (3), i.e. $a=0$, the integrand is proportional to $\left(k^{2}\right)^{-n}$ as $k^{2} \rightarrow 0$, as we have already mentioned. But $a \neq 0$ implies that the integrand behaves like

$$
\frac{1}{\left(\tilde{k}^{2}\right)^{n}\left[\frac{a^{\prime 2}}{k^{2}}\right]^{n+1}}=\frac{\tilde{k}^{2}}{\left(a^{\prime 2}\right)^{n+1}}
$$

which is independent of the loop order $n$. Using multiscale analysis, the perturbative renormalizability of this model to all orders could be shown [25].

\section{Noncommutative Gauge Theory}

The aim of this section is to generalize the approaches discussed above to noncommutative $U(1)$ gauge theory. They are good candidates for renormalizable models. As we will see, the UV/IR mixing also occurs in the case of noncommutative gauge theory, and so far, no model could be shown to be renormalizable.

\subsection{Oscillator approach}

As the first step, a BRST invariant action including an oscillator term has been proposed in [26]:

$$
\begin{aligned}
& S=\int d^{4} x\left(\frac{1}{4} F_{\mu \nu} \star F^{\mu \nu}+s\left(\bar{c} \star \partial_{\mu} A_{\mu}\right)-\right. \\
& \left.-\frac{1}{2} B^{2}+\frac{\Omega^{2}}{8} s\left(\tilde{c}_{\mu} \star \mathcal{C}_{\mu}\right)\right),
\end{aligned}
$$

where $\mathcal{C}_{\mu}$ contains the crucial new terms:

$\mathcal{C}_{\mu}=\left\{\left\{\tilde{x}_{\mu} \stackrel{\star}{,} A_{\nu}\right\} \stackrel{\star}{,} A_{\nu}\right\}+\left[\left\{\tilde{x}_{\mu} \stackrel{\star}{,} \bar{c}\right\}^{\star}, c\right]+\left[\bar{c} \stackrel{\star}{,}\left\{\tilde{x}_{\mu}, \stackrel{c}{,}\right\}\right]$,

and $\tilde{c}_{\mu}$ is a new parameter which also transforms under BRST. The noncommutative field strength is given by
$F_{\mu \nu}=\partial_{\mu} A_{\nu}-\partial_{\nu} A_{\mu}-\mathrm{i}\left[\mathrm{A}_{\mu} \stackrel{\star}{,} \mathrm{A}_{\nu}\right]$. Summing up, action (18) is invariant under the following BRST transformation:

$$
\begin{aligned}
& s A_{\mu}=D_{\mu} c, \quad s \bar{c}=B, \quad s c=i g c \star c, \\
& s B=0, \quad s \tilde{c}_{\mu}=\tilde{x}_{\mu} .
\end{aligned}
$$

The above set of transformations is nilpotent. The propagator of the gauge field is given by the Mehler kernel (13). One-loop calculations have been performed in [27]. A power counting formula has been obtained, and the corrections to the vertex functions have been computed. Remarkably, the one-point tadpole is UVdivergent. Therefore, action (18) is not stable under oneloop corrections, and linear counter terms are needed.

It seems natural to look for a more general action. The so-called induced gauge action $[28,29]$ contains the terms of (18) and more. It is invariant under noncommutative $U(1)$ transformations. The starting point is the scalar $\phi^{4}$ model with the oscillator potential (10). The scalar field is then coupled to an external gauge field. The dynamics of the gauge field is given by the divergent contributions of the one-loop effective action generalizing the method of heat kernel expansion to the noncommutative realm. The induced action is given by

$$
\begin{aligned}
& S=\int d^{4} x\left\{\frac{3}{\theta}\left(1-\rho^{2}\right)\left(\tilde{\mu}^{2}-\rho^{2}\right)\left(\tilde{X}_{\nu} \star \tilde{X}^{\nu}-\tilde{x}^{2}\right)+\right. \\
& \left.+\frac{3}{2}\left(1-\rho^{2}\right)^{2}\left(\left(\tilde{X}_{\mu} \star \tilde{X}_{\mu}\right)^{\star 2}-\left(\tilde{x}^{2}\right)^{2}\right)-\frac{\rho^{4}}{4} F_{\mu \nu} F_{\mu \nu}\right\},
\end{aligned}
$$

where $\rho=\frac{1-\Omega^{2}}{1+\Omega^{2}}, \tilde{\mu}^{2}=\frac{m^{2} \theta}{1+\Omega^{2}}$. Furthermore, the field strength is given by

$F_{\mu \nu}=-i\left[\tilde{x}_{\mu}, A_{\nu}\right]_{\star}+i\left[\tilde{x}_{\nu}, A_{\mu}\right]_{\star}-i\left[A_{\mu}, A_{\nu}\right]_{\star}$,

and $\tilde{X}_{\mu}$ denote the covariant coordinates, $\tilde{X}_{\mu}=\tilde{x}_{\mu}+A_{\mu}$. In the limit $\Omega \rightarrow 0$ (i.e., $\rho \rightarrow 1$ ), we recover the usual noncommutative Yang-Mills action. An interesting limit is $\Omega \rightarrow 1$ (i.e., $\rho \rightarrow 0$ ), where we obtain a pure matrix model. It has a nontrivial vacuum, which makes the quantization more difficult. The computation of propagators, construction of Feynman rules, and one-loop calculations are now in progress.

An alternative model has been proposed in [30]. The gauge model is constructed on a specific curved noncommutative background space, the so-called truncated Heisenberg space. In two dimensions, the action reads

$S=\int d^{2} x\left(\left(1-\alpha^{2}\right) F_{12}^{* 2}-2\left(1-\alpha^{2}\right) \mu F_{12} \star \phi+\right.$ 
$+\left(5-\alpha^{2}\right) \mu^{2} \phi^{2}+4 \mathrm{i} \alpha \mathrm{F}_{12} \star \phi^{\star 2}+$

$\left.+\left(D_{i} \phi\right)^{2}-\alpha^{2}\left\{p_{i}+A_{i} \stackrel{\star}{,} \phi\right\}^{2}\right)$,

where $\alpha$ is some parameter, and $\mu$ has dimension of a mass.

\section{2. $1 / p^{2}$ approach}

The same strategy as in 2.2 is applied here, and the IR divergence is added as a counter term. Considering the action

$S=\int d^{4} x F_{\mu \nu} \star F_{\mu \nu}$

for noncommutative $U(1)$ theory, the vacuum polarization shows the following IR divergent contribution:

$\Pi_{\mu \nu} \propto \frac{\tilde{p}_{\mu} \tilde{p}_{\nu}}{\left(\tilde{p}^{2}\right)^{2}}$.

A gauge invariant implementation of the above is given by the term [31]

$\int d^{4} x F_{\mu \nu} \frac{1}{\tilde{D}^{2} D^{2}} F_{\mu \nu}$.

The inverse covariant derivatives in the above expression should be expanded in terms of the gauge field. Hence, the vertices with an arbitrary number of photon legs occur. This situation might still be treatable, but it is simpler to use a localized version of (25). Basically, there are two different ways to implement the localization:

- By introducing an antisymmetric field $B_{\mu \nu}[32]$ :

$$
\begin{aligned}
& \int d^{4} x F_{\mu \nu} \frac{a^{2}}{\tilde{D}^{2} D^{2}} F_{\mu \nu} \rightarrow \\
& \rightarrow \int d^{4} x\left(a B_{\mu \nu} F_{\mu \nu}-B_{\mu \nu} \star \tilde{D}^{2} D^{2} B_{\mu \nu}\right) .
\end{aligned}
$$

But this field is physical and introduces additional degrees of freedom. Therefore, the model is not pure noncommutative $U(1)$ gauge theory any more, but describes different physics.

- Secondly, BRST doublet structures are employed in [33]. The additional fields are needed for the localization of the built BRST doublets (25). This avoids the introduction of new physical degrees of freedom. Unfortunately, the model presented in [33] is not renormalizable.
The virtue of the latter approach is the implementation of the IR damping as the so-called "soft breaking". This is in analogy to the Gribov-Zwanziger approach to undeformed QCD [34,35], where an IR modification of the propagator is suggested to cure the Gribov ambiguities. The UV renormalizability is not altered. In [36], the "soft breaking" approach has been developed further. As a result, the following action is proposed:

$S=S_{\mathrm{inv}}+S_{\mathrm{gf}}+S_{\mathrm{aux}}+S_{\mathrm{soft}}+S_{\mathrm{ext}}$,

$S_{\mathrm{inv}}=\int d^{4} x \frac{1}{4} F_{\mu \nu} F_{\mu \nu}$,

$S_{\mathrm{gf}}=\int d^{4} x s\left(\bar{c} \partial_{\mu} A_{\mu}\right)$

$S_{\mathrm{aux}}=\int d^{4} x s\left(\bar{\psi}_{\mu \nu} B_{\mu \nu}\right)$

$S_{\mathrm{soft}}=\int d^{4} x s\left(\left(\bar{Q}_{\mu \nu \alpha \beta} B_{\mu \nu}+Q_{\mu \nu \alpha \beta} \bar{B}_{\mu \nu}\right) \times\right.$

$\left.\times \frac{1}{\tilde{\square}}\left(f_{\alpha \beta}+\sigma \frac{\theta_{\alpha \beta}}{2} \tilde{f}\right)\right)$,

$S_{\mathrm{ext}}=\int d^{4} x\left(\Omega_{\mu}^{A} s A_{\mu}+\Omega^{c} s c\right)$

where $f_{\alpha \beta}=\partial_{\alpha} A_{\beta}-\partial_{\beta} A_{\alpha}$ is the commutative $U(1)$ field strength, $\Theta_{\alpha \beta}=\epsilon \theta_{\alpha \beta}, \tilde{f}=\theta_{\alpha \beta} f_{\alpha \beta}$, and $\tilde{\square}=\tilde{\partial}_{\mu} \tilde{\partial}_{\mu}=$ $\theta_{\mu \alpha} \theta_{\mu \beta} \partial_{\alpha} \partial_{\beta}$. For convenience, $\epsilon$ has mass dimension -2 , whereas $\theta_{\mu \nu}$ is rendered dimensionless. The additional sources $\bar{Q}, Q, \bar{J}, J$ ensure the BRST invariance of (27). In the IR, they take their physical values:

$\left.\bar{Q}_{\mu \nu \alpha \beta}\right|_{\mathrm{phys}}=0$,

$\left.\bar{J}_{\mu \nu \alpha \beta}\right|_{\mathrm{phys}}=\frac{\gamma^{2}}{4}\left(\delta_{\mu \alpha} \delta_{\nu \beta}-\delta_{\mu \beta} \delta_{\nu \alpha}\right)$

$\left.Q_{\mu \nu \alpha \beta}\right|_{\mathrm{phys}}=0$,

$\left.J_{\mu \nu \alpha \beta}\right|_{\mathrm{phys}}=\frac{\gamma^{2}}{4}\left(\delta_{\mu \alpha} \delta_{\nu \beta}-\delta_{\mu \beta} \delta_{\nu \alpha}\right)$. 
Inserting the physical values and integrating out the field $B_{\mu \nu}$, the following action is obtained:

$$
\begin{aligned}
& S_{\text {phys }}=\int d^{4} x\left(\frac{1}{4} F_{\mu \nu} F_{\mu \nu}+\gamma^{4}\left[\partial_{\mu} A_{\nu} \frac{1}{2 \widetilde{\square}^{2}} f_{\mu \nu}+\right.\right. \\
& \left.\left.+\left(\sigma+\frac{\theta^{2}}{4} \sigma^{2}\right)(\tilde{\partial} A) \frac{1}{\widetilde{\square}^{2}}(\tilde{\partial} A)\right]+s\left(\bar{c} \partial_{\mu} A_{\mu}\right)\right) .
\end{aligned}
$$

The term proportional to $\gamma^{4}$ breaks the gauge invariance. It is called the "soft breaking," since the parameter $\gamma$ has dimension of mass. We have used the commutative field strength in this expression, although it is not covariant under noncommutative gauge transformations. But it only appears in the breaking term and cannot make it worse, since the gauge invariance is already violated. The advantage is that only the propagation, but not the interaction, is modified due to the "soft breaking."

The full action (27) is invariant under the following set of BRST transformations:

$$
\begin{aligned}
& s A_{\mu}=D_{\mu} c, \quad s c=i g c c, \quad s \bar{c}=b, \quad s b=0, \\
& s \bar{\psi}_{\mu \nu}=\bar{B}_{\mu \nu}, \quad s \bar{B}_{\mu \nu}=0 \\
& s B_{\mu \nu}=\psi_{\mu \nu}, \quad s \psi_{\mu \nu}=0, \\
& s \bar{Q}=\bar{J}, \quad s \bar{J}=0, \quad s Q=J, \quad s J=0 .
\end{aligned}
$$

The fields $\psi$ and $B$, respectively, $\bar{\psi}$ and $\bar{B}$, and the sources $Q$ and $J$, respectively, $\bar{Q}$ and $\bar{J}$, are BRST doublets. Let us discuss the Feynman rules for (27). The vertex functions are the same as in the usual noncommutative $U(1)$ theory defined by action (23). The propagator is more complicated; it reads

$$
\begin{aligned}
& G_{\mu \nu}^{A}(k)=\left(k^{2}+\frac{\gamma^{4}}{\tilde{k}^{2}}\right)^{-1} \times \\
& \times\left(\delta_{\mu \nu}-\frac{k_{\mu} k_{\nu}}{k^{2}}-\frac{\bar{\sigma}^{4}}{\left(k^{2}+\left(\bar{\sigma}^{4}+\gamma^{4}\right) \frac{1}{\tilde{k}^{2}}\right)} \frac{\tilde{k}_{\mu} \tilde{k}_{\nu}}{\left(\tilde{k}^{2}\right)^{2}}\right),
\end{aligned}
$$

where

$$
\bar{\sigma}=2 \gamma^{4}\left(\sigma+\frac{\theta^{2} \sigma^{2}}{4}\right)
$$

But, in 1-loop calculations, it can be approximated by

$G_{\mu \nu}^{A} \sim \frac{1}{k^{2}}\left(\delta_{\mu \nu}-\frac{k_{\mu} k_{\nu}}{k^{2}}\right), \quad k^{2} \gg 1$ since both UV and IR divergences result from the highmomentum range in the loop. This ignores the IR damping, but, as we have seen, the damping has no effect at the one-loop level. Considering higher-loop insertions of a single tadpole (cf. (16)), the damping of the propagators between the single loops is essential and renders the result independent of the number of inserted loops at least in the scalar case. For the gauge model discussed here, this still needs to be shown.

A power counting formula,

$d_{G}=4-E_{A}-E_{c \bar{c}}$,

where $E_{\phi}$ denotes the number of external $\phi$-legs, and one-loop results have been obtained in [36]. The correction to the vacuum polarization is given by

$\Pi_{\mu \nu}=\frac{2 g^{2}}{\epsilon^{2} \pi^{2}} \frac{\tilde{p}_{\mu} \tilde{p}_{\nu}}{\left(\tilde{p}^{2}\right)^{2}}+\frac{13 g^{2}}{3(4 \pi)^{2}}\left(p^{2} \delta_{\mu \nu}-p_{\mu} p_{\nu}\right) \ln \Lambda$

where $\Lambda$ denotes a momentum cut-off. Remarkably, the one-loop correction is transversal. Furthermore, we obtained the following results for the vertices:

$$
\begin{aligned}
& \Gamma_{\mu \nu \rho}^{3 \mathrm{~A}, \mathrm{IR}}=-\frac{2 i g^{3}}{\pi^{2}} \cos \frac{\epsilon p_{1} \tilde{p}_{2}}{2} \sum_{j=1,2,3} \frac{\tilde{p}_{j, \mu} \tilde{p}_{j, \nu} \tilde{p}_{j, \rho}}{\epsilon\left(\tilde{p}_{j}^{2}\right)^{2}}, \\
& \Gamma_{\mu \nu \rho}^{3 \mathrm{~A}, \mathrm{UV}}=-\frac{17 g^{2}}{6(4 \pi)^{2}} \ln \Lambda \tilde{V}_{\mu \nu \rho}^{3 \mathrm{~A}, \text { tree }}\left(p_{1}, p_{2}, p_{3}\right),
\end{aligned}
$$

$\Gamma_{\mu \nu \rho \sigma}^{4 \mathrm{~A}, \mathrm{UV}}=-\frac{5}{8 \pi^{2}} \ln \Lambda \tilde{V}_{\mu \nu \rho \sigma}^{4 \mathrm{~A}, \text { tree }}$,

where $V_{\mu \nu \rho}^{3 \mathrm{~A}, \text { tree }}$ and $V_{\mu \nu \rho \sigma}^{4 \mathrm{~A}, \text { tree }}$ denote the tree level vertex functions. Regarding the three-point function, the IR divergent result (40) corresponds to a counter term

$S^{3 \mathrm{~A}, \text { corr }}=\int d^{4} x g^{3}\left\{A_{\mu} \stackrel{\star}{,} A_{\nu}\right\} \frac{\tilde{\partial}_{\mu} \tilde{\partial}_{\nu} \tilde{\partial}_{\rho}}{\epsilon \tilde{\square}^{2}} A_{\rho}$.

Such a term can readily be introduced into the "soft breaking" part of the action $S_{\text {soft }}$ in (27). But in order to do so, we have to restore the BRST invariance in the UV regime. Again, this can be achieved by introducing sources $Q^{\prime}$ and $J^{\prime}$, which form a BRST doublet,

$s Q^{\prime}=J^{\prime}, \quad s J^{\prime}=0$.

Consequently, we insert the following terms into $S_{\text {soft }}$ :

$\int d^{4} x\left(J^{\prime}\left\{A_{\mu}, A_{\nu}\right\} \frac{\tilde{\partial}_{\mu} \tilde{\partial}_{\nu} \tilde{\partial}_{\rho}}{\tilde{\square}^{2}} A_{\rho}-\right.$ 
$\left.-Q^{\prime} s\left(\left\{A_{\mu} \stackrel{\star}{,} A_{\nu}\right\} \frac{\tilde{\partial}_{\mu} \tilde{\partial}_{\nu} \tilde{\partial}_{\rho}}{\tilde{\square}^{2}} A_{\rho}\right)\right)$.

This term is BRST-invariant by itself. In the IR, the sources take on their physical values

$J^{\prime}=g \gamma^{\prime 2}, \quad Q^{\prime}=0$,

and the counter term in (43) leads to a renormalization of $\gamma^{\prime}$, which is another parameter of mass-dimension 1 .

The above one-loop result leads to the negative $\beta$ function:

$\beta=-\frac{7 g^{3}}{12 \pi^{2}}$.

The commutative $\beta$-function is positive. From this equation alone, it becomes clear that there is no smooth transition to the undeformed physics on a quantum level.

\section{Concluding Remarks}

The one-loop corrections for the novel action (27) reduce to the ones known from the usual noncommutative $U(1)$ theory (see, e.g., $[18,37])$. At higher-loop orders, differences will arise. Both, UV and IR divergences can be absorbed in the tree level action (27) plus (45). But so far, a renormalization (dis)proof is still missing. We plan to attack this problem by applying a renormalization scheme such as multiscale analysis or flow equations. The negative $\beta$-function reflects the non-Abelian structure of noncommutative $U(1)$ gauge theory.

Concerning the induced gauge action (21), we plan to study the vacuum structure, to study its quantization, and, as a first step, to compute one-loop corrections.

1. E. Schrödinger, Die Naturwiss. 31, 518 (1934).

2. W. Heisenberg, Ann. Phys. 32, 20 (1938).

3. S. Doplicher, K. Fredenhagen, and J.E. Roberts, Commun. Math. Phys. 172, 187 (1995), http://www.arXiv.org/ abs/hep-th/0303037.

4. L.J. Garay, Int. J. Mod. Phys. A 10, 145 (1995), http://arXiv.org/abs/gr-qc/9403008.

5. A. Connes, M. R. Douglas, and A. S. Schwarz, JHEP 02, 003 (1998), http://www.arXiv.org/abs/hep-th/9711162.

6. N. Seiberg and E. Witten, JHEP 09, 032 (1999); http://arXiv.org/abs/hep-th/9908142.

7. V. Schomerus, JHEP 06, $030 \quad$ (1999), http://www.arXiv.org/abs/hep-th/9903205.

8. A. Connes, Inst. Hautes Etudes Sci. Publ. Math. 62, 257 (1986).
9. J. Bellissard, Ordinary quantum Hall effect and noncommutative cohomology. Lecture given at the Conference on Localization in Disordered System, Bad Schandau, DDR, Dec 1-5, 1986.

10. R. J. Szabo, Class. Quant. Grav. 23, R199 (2006), http://www.arXiv.org/abs/hep-th/0606233.

11. H. S. Yang, Int. J. Mod. Phys. A 24, 4473 (2009), http://www.arXiv.org/abs/hep-th/0611174.

12. X. Calmet and A. Kobakhidze, Phys. Rev. D 74, 047702 (2006), http://www.arXiv.org/abs/hep-th/0605275.

13. H. Steinacker, JHEP 12, 049 (2007), http://www.arXiv.org/abs/arXiv:0708.2426 [hep-th].

14. M. Chaichian, A. Tureanu, and G. Zet, Phys. Lett. B 660, 573 (2008), http://www.arXiv.org/abs/0710.2075.

15. A. P. Balachandran, A. Pinzul, B. A. Qureshi, and S. Vaidya, Phys. Rev. D 76, 105025 (2007), http://www.arXiv.org/abs/arXiv:0708.0069 [hep-th].

16. S. Minwalla, M. Van Raamsdonk, and N. Seiberg, JHEP 02, 020 (2000), http://www.arXiv.org/abs/hepth/9912072.

17. I.Y. Aref'eva, D.M. Belov, A.S. Koshelev, and O.A. Rytchkov, Nucl. Phys. Proc. Suppl. 102, 11 (2001), http://www.arXiv.org/abs/hep-th/0003176.

18. A. Matusis, L. Susskind, and N. Toumbas, JHEP 12, 002 (2000), http://www.arXiv.org/abs/hep-th/0002075.

19. A. Micu and M.M. Sheikh Jabbari, JHEP 01, 025 (2001), http://www.arXiv.org/abs/hep-th/0008057.

20. J.M. Grimstrup, H. Grosse, L. Popp, V. Putz, M. Schweda, M. Wickenhauser, and R. Wulkenhaar, Europhys. Lett. 67, 186 (2004), http://www.arXiv.org/ abs/hep-th/0202093.

21. H. Grosse and R. Wulkenhaar, JHEP 12, 019 (2003), http://www.arXiv.org/abs/hep-th/0307017.

22. H. Grosse and R. Wulkenhaar, Commun. Math. Phys. 256, 305 (2005), http://www.arXiv.org/abs/hepth/0401128.

23. E. Langmann and R.J. Szabo, Phys. Lett. B 533, 168 (2002), http://www.arXiv.org/abs/hep-th/0202039.

24. M. Buric and M. Wohlgenannt, JHEP 03, 053 (2010), http://www.arXiv.org/abs/0902.3408.

25. R. Gurau, J. Magnen, V. Rivasseau, and A. Tanasa, Commun. Math. Phys. 287, 275 (2009), http://www.arXiv.org/ abs/0802.0791.

26. D. N. Blaschke, H. Grosse, and M. Schweda, Europhys. Lett. 79, 61002 (2007), http://www.arXiv.org/abs/ 0705.4205 .

27. D.N. Blaschke, H. Grosse, E. Kronberger, M. Schweda, and M. Wohlgenannt, http://www.arXiv.org/abs/ 0912.3642 .

28. A. de Goursac, J.-C. Wallet, and R. Wulkenhaar, Eur. Phys. J. C 51, 977 (2007), http://www.arXiv.org/ abs/hep-th/0703075. 
29. H. Grosse and M. Wohlgenannt, Eur. Phys. J. C 52, 435 (2007), http://www.arXiv.org/abs/hep-th/0703169.

30. M. Buric, H. Grosse, and J. Madore, JHEP 07, 010 (2010), http://www.arXiv.org/abs/1003.2284.

31. D.N. Blaschke, F. Gieres, E. Kronberger, M. Schweda, and M. Wohlgenannt, J. Phys. A 41, 252002 (2008), http://www.arXiv.org/abs/0804.1914.

32. D.N. Blaschke, A. Rofner, and R.I.P. Sedmik, SIGMA 6, 037 (2010), http://www.arXiv.org/abs/0908.1743.

33. L.C.Q. Vilar, O.S. Ventura, D.G. Tedesco, and V.E.R. Lemes, J. Phys. A 43, 135401 (2010).

34. D. Zwanziger, Nucl. Phys. B 323, 513 (1989).

35. D. Zwanziger, Nucl. Phys. B 399, 477 (1993).

36. D.N. Blaschke, A. Rofner, R.I.P. Sedmik, and M. Wohlgenannt, http://www.arXiv.org/abs/0912.2634.
37. M. Hayakawa, Phys. Lett. B 478, 394 (2000), http://arXiv.org/abs/hep-th/9912094.

Received 15.07.11

\section{НЕКОМУТАТИВНІ КАЛІБРУВАЛЬНІ ТЕОРІї}

M. Волгенант

Р ез юм е

Розглянуто два різних підходи, які узагальнюють калібрувальні моделі до перенормованих скалярних квантових теорій поля. Один $з$ них реалізує критичне інфрачервоне затухання пропагатора калібрувального поля в так званій частині “м'якого розриву". Також обговорено однопетлеве перенормування. 